\title{
Strongly enhanced shot noise in chains of quantum dots
}

\author{
Jasmin Aghassi, ${ }^{1,2}$ Axel Thielmann, ${ }^{1,2}$ Matthias H. Hettler, ${ }^{1}$ and Gerd Schön ${ }^{1,2}$ \\ ${ }^{1}$ Forschungszentrum Karlsruhe, Institut für Nanotechnologie, 76021 Karlsruhe, Germany \\ ${ }^{2}$ Institut für Theoretische Festkörperphysik, Universität Karlsruhe, 76128 Karlsruhe, Germany
}

(Dated: February 2, 2008)

\begin{abstract}
We study charge transport through a chain of quantum dots. The dots are fully coherent among each other and weakly coupled to metallic electrodes via the dots at the interface, thus modelling a molecular wire. If the non-local Coulomb interactions dominate over the inter-dot hopping we find strongly enhanced shot noise above the sequential tunneling threshold. The current is not enhanced in the region of enhanced noise, thus rendering the noise super-Poissonian. In contrast to earlier work this is achieved even in a fully symmetric system. The origin of this novel behavior lies in a competition of "slow" and "fast" transport channels that are formed due to the differing non-local wave functions and total spin of the states participating in transport. This strong enhancement may allow direct experimental detection of shot noise in a chain of lateral quantum dots.
\end{abstract}

PACS numbers: 73.63.-b, 73.23.Hk, 72.70.+m

Introduction- Shot noise in mesoscopic devices such as point contacts and quantum dots has been the subject of intensive research within the last decade 1]. Recently the interest has been intensified both theoretically $[2,3,4,5,6,7,8,9]$ and experimentally [10, 11, 12] when the importance of shot noise for quantum computing, nanomechanical systems and molecular devices had been recognized. However, experiments on these systems are difficult to perform, since one needs to detect the shot noise over the background of $1 / f$ - noise caused by fluctuations in the physical environment and measurement equipment. So far even for lateral quantum dots in semiconductor heterostructures, with far reaching control of system parameters and advanced low temperature setups, no direct shot noise measurements have been reported [12].

In an array of quantum dots with Coulomb interactions, both the current $I$ and the shot noise $S$ depend on details of the electronic spectrum of the many-body states of the coupled system, as well as the coupling strengths of these states to the electrodes [4, 6]. Thus the combined measurements of current and shot noise provide a 'spectroscopic' tool to gain information about the level structure. For 'local' systems such as single dots or two parallel dots (which can be considered as a single dot with two or more single-particle levels) the shot noise is known to be mostly sub-Poissonian $(S<2 e I$. SuperPoissonian noise is predicted in asymmetric situations, with level- or spin-dependent couplings to the quantum dot $3,[4,6]$, or sometimes in the Coulomb blockade region [8, 13]. Similar considerations hold for two dots in series [14].

In this letter we show that starting with a chain of three dots the noise may become super-Poissonian even if the dot-electrode couplings are fully symmetric (spatial and spin symmetry). The shot noise is greatly enhanced in absolute magnitude due to a strong competition between "slow" and "fast" transport channels, if the non-local Coulomb repulsion dominates the interdot hopping. As there is no simultaneous enhancement of the tunneling current, the shot noise becomes superPoissonian. Such behavior can be achieved involving only electronic degrees of freedom, and over a large bias region above the sequential tunneling threshold. The strong enhancement of the shot noise in a chain of lateral quantum dots should allow its direct experimental detection.

Model and technique- We consider a series of quantum dots, each with one spin-degenerate level. Including hopping between the dots as well as intra-dot and interdot (nearest neighbor) Coulomb interactions we arrive at the Hamiltonian $\hat{H}=\hat{H}_{\mathrm{L}}+\hat{H}_{\mathrm{R}}+\hat{H}_{\text {dots }}+\hat{H}_{\mathrm{T}, \mathrm{L}}+\hat{H}_{\mathrm{T}, \mathrm{R}}$ with

$$
\begin{aligned}
\hat{H}_{r}= & \sum_{\mathbf{k} \sigma} \epsilon_{\mathbf{k}} a_{\mathbf{k} \sigma r}^{\dagger} a_{\mathbf{k} \sigma r}, \hat{H}_{\mathrm{T}, r}=\sum_{i \mathbf{k} \sigma}\left(t_{i}^{r} a_{\mathbf{k} \sigma r}^{\dagger} c_{i \sigma}+\text { h.c. }\right), \\
\hat{H}_{\mathrm{dots}} & =\sum_{i \sigma} \epsilon_{i} n_{i \sigma}-t \sum_{<i j>\sigma}\left(c_{i \sigma}^{\dagger} c_{j \sigma}+h . c .\right) \\
& +U \sum_{i} n_{i \uparrow} n_{i \downarrow}+U_{n n} \sum_{<i j>\sigma \sigma^{\prime}} n_{i \sigma} n_{j \sigma^{\prime}},
\end{aligned}
$$

where $i=1 \ldots N$ and $r=\mathrm{L}, \mathrm{R}$. Here, $\hat{H}_{\mathrm{L}}$ and $\hat{H}_{\mathrm{R}}$ model the non-interacting electrons with density of states $\rho_{e}$ (assumed as a constant) in the left and right electrode described by the Fermi operators $a_{\mathbf{k} \sigma r}^{\dagger}, a_{\mathbf{k} \sigma r}$. The "artificial molecule" term $\hat{H}_{\text {dots }}$ describes the series of dots with Fermi operators $c_{i \sigma}^{\dagger}, c_{i \sigma}$, on-site energies $\epsilon_{i}$ and nearest neighbor hopping $t$. $U$ and $U_{n n}$ are the strength of the intra-dot and nearest neighbor inter-dot Coulomb repulsion. Tunneling between the leads and dots is modeled by $\hat{H}_{\mathrm{T}, \mathrm{L}}$ and $\hat{H}_{\mathrm{T}, \mathrm{R}}$. We restrict ourselves to situations where the leads couple only to the one adjacent dot. The coupling strength is characterized by $\Gamma_{i}^{r}=2 \pi\left|t_{i}^{r}\right|^{2} \rho_{e}$, where $t_{i}^{r}$ are the tunneling matrix elements.

We are interested in transport through a chain of quantum dots, in particular in the current $I$ and the (zero-frequency) current noise $S$. They are related to 
the current operator $\hat{I}=\left(\hat{I}_{R}-\hat{I}_{\mathrm{L}}\right) / 2$, with $\hat{I}_{r}=$ $-i(e / \hbar) \sum_{i \mathbf{k} \sigma}\left(t_{i}^{r} a_{\mathbf{k} \sigma r}^{\dagger} c_{i \sigma}-h . c.\right)$ being the current operator for electrons tunneling into lead $r$, by $I=\langle\hat{I}\rangle$ and

$$
S=\int_{-\infty}^{\infty} d t\langle\delta \hat{I}(t) \delta \hat{I}(0)+\delta \hat{I}(0) \delta \hat{I}(t)\rangle
$$

where $\delta \hat{I}(t)=\hat{I}(t)-\langle\hat{I}\rangle$.

For the calculation of the current $I$ and current noise $S$, we use the diagrammatic technique on the Keldysh contour developed in Ref. 15 and expanded for the description of the noise in Ref. 6. In this approach the dot array $\left(H_{\text {dots }}\right)$ is treated exactly as an interacting many-body system, but is perturbatively (incoherently) coupled to the electrodes. We diagonalize the dot Hamiltonian $H_{\text {dots }}$ and compute the transition rates $W_{\chi \chi^{\prime}}$ (forming a matrix W) between two eigenstates $\chi$ and $\chi^{\prime}$ in first order perturbation theory in the coupling strengths $\Gamma_{i}^{r}$. Given the transition rates $\mathbf{W}$, the stationary probabilities $\mathbf{p}^{\text {st }}$ (the diagonal elements of the reduced density matrix of the dot array) can be found from a master equation. By making use of the conservation of probability, we rewrite the master equation in the form $\tilde{\mathbf{W}} \mathbf{p}^{\text {st }}=\mathbf{v}$, where $\tilde{\mathbf{W}}$ is identical to $\mathbf{W}$ but with one (arbitrarily chosen) row $\chi_{0}$ being replaced with $(\Gamma, \ldots, \Gamma)$. The vector $\mathbf{v}$ is defined as $v_{\chi}=\Gamma \delta_{\chi \chi_{0}}$. The current and shot noise are given by:

$$
\begin{gathered}
I=\frac{e}{2 \hbar} \mathbf{e}^{T} \mathbf{W}^{I} \mathbf{p}^{\mathrm{st}} \\
S=\frac{e^{2}}{\hbar} \mathbf{e}^{T}\left(\mathbf{W}^{I I} \mathbf{p}^{\mathrm{st}}+\mathbf{W}^{I} \mathbf{P} \mathbf{W}^{I} \mathbf{p}^{\mathrm{st}}\right) .
\end{gathered}
$$

The vector $\mathbf{e}$ is defined as $e_{\chi}=1$ for all $\chi$ and the matrices $\mathbf{W}^{I}\left(\mathbf{W}^{I I}\right)$ denote the transition rates with one (two) current vertex replacing one (two) tunneling vertex due to $H_{\mathrm{T}, \mathrm{L}}$ or $H_{\mathrm{T}, \mathrm{R}}$. The "propagator" $\mathbf{P}$ is obtained from $\tilde{\mathbf{W}} \mathbf{P}=\mathbf{Q}$ with $Q_{\chi^{\prime} \chi}=\left(p_{\chi^{\prime}}^{\text {st }}-\delta_{\chi^{\prime} \chi}\right)\left(1-\delta_{\chi^{\prime} \chi_{0}}\right)$. Further details of the technique can be found in Ref. 6 .

Note that the noise expression Eq. 4 consists of two terms: The first term is associated with $\mathbf{W}^{I I}$, i.e. noise diagrams with two current vertices in a single irreducible block. This term typically gives a noise contribution of $e I$. The second term is due to reducible noise diagrams. It involves the "propagator" $\mathbf{P}$ of the dot system and therefore accounts for the electronic structure and the correlations on the central system. It is the second term that is responsible for the interesting correlation physics dicussed below.

Results- In the following we discuss current and shot noise for a model of type Eqs. [1 with $N=3$ dots and a half filled ground state (i.e. one electron per dot). We use equal couplings, $\Gamma_{1}^{L}=\Gamma_{3}^{R}=2.5 \mu \mathrm{eV}$, so $\Gamma=\Gamma_{1}^{L}+\Gamma_{3}^{R}=$ $5 \mu \mathrm{eV}$. Our perturbation expansion is valid for temperatures much larger than the tunnel couplings. Throughout this paper, we choose $k_{\mathrm{B}} T=5 \Gamma=0.025 \mathrm{meV}$ which corresponds to $T \approx 0.25 \mathrm{~K}$. The dot system is characterized by the (uniform) level energy $\epsilon$, the intra-dot 'Hubbard' repulsion $U$ and the nearest neighbor charge repulsion $U_{n n}$, which we present in units of meV. Transport is achieved by applying a bias voltage $V_{\mathrm{b}}$, which is dropped symmetrically and entirely at the electrode-dot tunnel junctions, meaning that the energies of the dot states are independent of the applied voltage. The effects of polarization due to asymmetric or incomplete voltage drops are straightforward to anticipate, but would only add unnecessary complexity to the results presented below.

We point out that the three dot system is the simplest system that is not pure interface and therefore is the minimal model for a truly non-local "artificial molecule". States which are mainly "localized" at the interfacial dots are competing in transport with states that have their main weight at the middle dot (even though the Hamiltonian remains fully left $\leftrightarrow$ right symmetric). This competition can have dramatic effects in the noise characteristics that have no equivalent for smaller systems.

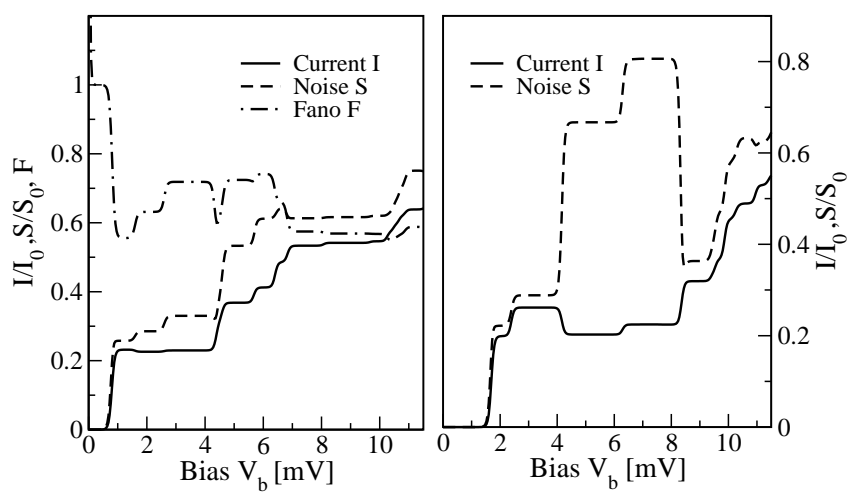

FIG. 1: Left panel: current $I$, shot noise $S$ and Fano factor $F$ vs. bias voltage for a three-dot chain with $k_{\mathrm{B}} T=0.025$, $\epsilon=-10, t=2, U=12$ and $U_{n n}=0.2$ (all energies in units of meV). The current increases stepwise while the noise is sub-Poissonian for the all bias larger than the sequential tunneling threshold. Right panel: current $I$ and noise $S$ for same parameters as on the left, except that $\epsilon=-10.8, U_{n n}=$ 5. Above $V_{b} \sim 4 \mathrm{mV}$ a region of strongly enhanced noise appears, whereas the current behaves in a similar manner as in the left panel. All current and noise curves are normalized to $I_{0}=(e / 2 \hbar) \Gamma \sim 60 \mathrm{pA}$ and $S_{0}=\left(e^{2} / 2 \hbar\right) \Gamma \sim 10^{-29} \mathrm{~A}^{2} / \mathrm{Hz}$, respectively.

We first consider a situation where the non-local interaction $U_{n n}$ is small $\left(U_{n n}=t / 10\right)$ and obtain the typical behavior for a fully symmetric system, see left panel of Fig. 1. The current rises (mostly) monotonically in steps, the noise also shows steps, but must not increase monotonically (thermally broadened peaks around the steps are also possible). The Fano factor $F=S / 2 e I$ will fall between values of 1 (Poissonian noise) and $1 / 2$ for biases larger than all excitation energies (symmetric double barrier noise), though in general it will not fall 
with a monotonous dependence on bias. At small bias, $e V_{b} \ll k_{B} T$, the noise is dominated by thermal noise, leading to a divergence [2] of the Fano factor.

If now the non-local Coulomb repulsion $U_{n n}$ is increased so that $U_{n n}>t$ we observe (right panel of Fig. 1) the usual stepwise increase of current and noise except for a bias region beyond the sequential tunneling threshold, where the noise is strongly enhanced, accompanied with a decrease in the current, signaling negative differential conductance (NDC). The noise enhancement (and NDC) is the result of the occupation of the quadruplet state $Q$ (see inset of Fig. 2 and discussion below) that, due to the nature of its wave function, contributes a "slow" channel of transport competing with other "fast" channels.

It is important to note that the strong enhancement of the noise does not require NDC. As an example, in Fig. 2 we show current, noise and Fano factor for the same parameters as for Fig. 1 right panel, except for a different $\epsilon=-10$. The current shows generic behavior, i.e. stepwise increase and only a tiny NDC around $V_{b}=8.5 \mathrm{mV}$. The noise, however, is tremendously enhanced, with the Fano factor $F>1$ indicating its super-Poissonian nature. While $F>1$ in itself is not uncommon in the sequential tunneling regime [3, [4, [] ], often $F>1$ is achieved by a suppression of transport in which the current is more suppressed than the noise. Here, the current is not suppressed, but the noise itself is enhanced in absolute magnitude over a large bias range, before recovering "normal" behavior beyond a bias of $V_{b}=10 \mathrm{mV}$.

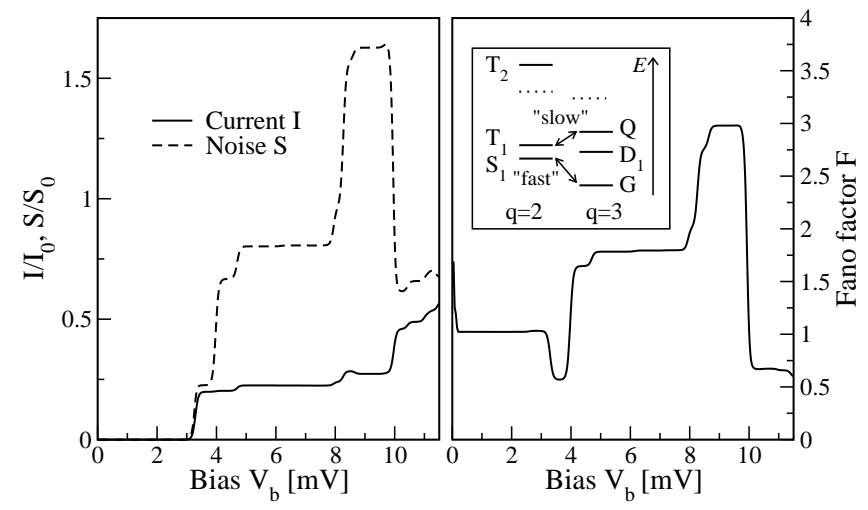

FIG. 2: Current $I$ and shot noise $S$ vs. voltage for three dots $k_{\mathrm{B}} T=0.025, t=2, \epsilon=-10, U=12, U_{n n}=5$. The noise is strongly enhanced in absolute magnitude above $V_{b} \sim 4 \mathrm{mV}$, while the current only slightly increases, leading to a Fano factor $F>1$. This is due to a competition of "fast" and "slow" transport channels. The noise scales like $\left(U_{n n} / t\right)^{2}$ in this regime, while the current saturates as $t$ is lowered. Above $V_{b} \sim 10 \mathrm{mV}$ "normal" behavior resumes, as the "slow" channel of transport is "cut short" (see text).

For such a noise behavior, firstly, strong electron interactions $\left(U, U_{n n} \gg T\right)$ are needed to have the various states compete in transport. Secondly, the outcome of this competition is determined by the wave functions of the competing states that effectively generate state dependent tunneling transition rates. Finally, the total spin of the states in question can differ by more than the electron spin $1 / 2$, so some energetically and spatially possible transition rates vanish due to spin selection rules. In the present case, the dominance of the non-local interaction $U_{n n}$ over the hopping $t$, leads to a strong modification in the spatial distribution of the relevant many-body wave functions as compared to the $U_{n n} \ll t$ case. Let us consider the 9 states with total spin 1 in the $q=2$ charge sector that split into three triplets (only $T_{1}, T_{2}$ shown in the insert of Fig. 2 If $U_{n n}<t$, the lowest triplet $\left(T_{1}\right)$ will prefer to have electrons on the middle dot, to maximize the kinetic energy. On the other hand, if $U_{n n}>t$ it prefers to have one electron each on the leftmost and the rightmost dot, thus minimizing both intra-dot and interdot Coulomb repulsion. This change in the nature of the lowest triplet wave function is crucial for the "noisy" transport. An equally fundamental role plays the total spin $3 / 2$ quadruplet, the second excited state in the $q=3$ charge sector. Due to spin selection rules these quadruplet states can only have tunneling transitions to the triplet states of the $q=2$ (or $q=4$ ) charge sectors. The transitions between the lowest triplet and the quadruplet form a "slow" channel of transport. The transition rate between them is suppressed by a factor $\sim\left(t / U_{n n}\right)^{2}$, as the tunneling can happen only at the interface dots, but the wave functions of the triplet and quadruplet mainly differ by an electron in the middle dot. The current effectively alternates between "fast" tunneling sequences between the well connected doublet $\left(D_{1}, G\right)$ and singlet states $\left(S_{1}\right)$, and the "slow" sequences between the lowest triplet $\left(T_{1}\right)$ and quadruplet state $(Q)$, leading to the super-Poissonian noise in the corresponding bias regime.

In Fig. [2] on the first plateau for $3.3 \mathrm{mV}<V_{b}<4 \mathrm{mV}$ with sub-Poissonian noise, transport is mainly achieved by tunneling events in which the dot system alternates between the ground state $G$ with charge $q=3$, and the lowest singlet $S_{1}$ with charge $q=2$. The states $S_{1}$ and the lowest triplet $T_{1}(q=2)$ are only split by the small "exchange energy" of size $J \sim t^{2} / U \sim 0.33 \mathrm{meV}$ and the first excited doublet $D_{1}(q=2)$ falls energetically in between $S_{1}$ and $T_{1}$, see the inset in the right panel of Fig. 2 Therefore, as soon as the sequential tunneling threshold $V_{\text {seq }}=2\left(E_{S_{1}}-E_{G}\right) / e \sim 3.3 \mathrm{mV}$ is overcome, the states $T_{1}$ and $D_{1}$ also participate in transport, though their occupation turns out to be small (less than 1 percent). Since $V_{\text {seq }}>2\left(E_{Q}-E_{T_{1}}\right) / e$ even the quadruplet $Q$ is occupied, though with the same low probability as the triplet $T_{1}$ [16]. However, above $V_{b}=2\left(E_{T_{1}}-E_{G}\right) / e=4 \mathrm{mV}$ the transport becomes noisy, with a Fano factor $F>1$, though the current itself still increases by about 2 percent (not visible in the figure). When direct transitions between the ground state $G$ and lowest triplet $T_{1}$ become possible, the occupation in $T_{1}, D_{1}$ and $Q$ increases at the cost of $G$ and $S_{1}$, until each state has equal probability. 
Due to their larger spin multiplicity this means that the triplet and the quadruplet states compose about $7 / 12$ of the total probability. For even larger bias, states in the $q=4$ charge sector come into play, which leads to several smaller features, until at about $V_{b}=8 \mathrm{mV}$ the quadruplet becomes even more occupied via the lowest triplet in the $q=4$ charge sector and the noise and the Fano factor reach their maximum. The maximum value of noise (and Fano factor) behave as $\left(U_{n n} / t\right)^{2}$, whereas the current value at the corresponding plateau saturates as the hopping $t$ is decreased. This is due to the homogeneity of our Hamiltonian: site disorder, i.e. dot-dependent $\epsilon$, will lead to a decreasing current with decreasing $t[\underline{5}$. The size of $t$, however, is restricted to the condition $t \gg \Gamma^{r}$, which is needed for the division of the total system into a coherent dot system with perturbative coupling to electrodes to make sense.

At $V_{b}=10 \mathrm{mV}$ transitions between the quadruplet and the second triplet $\left(T_{2}\right)$ with $q=2$ become possible. In contrast to the lowest triplet $T_{1}$, the states of the second triplet (as well as the quadruplet states) do not benefit from the hopping $t$, therefore the structure of their wave functions is independent of both the interactions $U, U_{n n}$ and the hopping $t$, only the actual energy varies $\left(E_{T_{2}}=\right.$ $\left.2 \epsilon+U_{n n}, E_{Q}=3 \epsilon+2 U_{n n}\right)$. The second triplet is spread uniformly over the three dots, its transition rate with the quadruplet is not suppressed with $t / U_{n n}$. Therefore, the slow channel between the $T_{1}$ triplet and the quadruplet is "cut short", the transport becomes more homogeneous and the Fano factor drops below unity.

We conclude with a few remarks: 1) As the above effect relies on spin quantum numbers we believe that it will be robust to standard relaxation processes involving phonon and photon emission. A strong magnetic field will modify the details, but not the generic behavior of the transport. 2) With a change of $\epsilon$ (gate voltage), i.e. $\epsilon=-11.5$, the enhancement of shot noise can be achieved even at the first plateau. Although the details of how each state exactly contributes to the transport at a given bias are changed, the main mechanism (the slow tripletquadruplet channel) to the enhanced noise is the same. 3) For $U_{n n} \ll t$ a similar spatial distribution of the lowest triplet wave function can be achieved by raising the level position $\epsilon_{2}$ of the middle dot more than $t$ above the $\epsilon$ of the interfacial dots. However, such a level detuning will strongly reduce both current and shot noise, similar to the "local" models of Refs. 4, 6. 4) In the Coulomb blockade region and close to the sequential tunneling threshold we often find super-Poissonian noise. This enhancement is due to the thermal occupation and following sequential depletion of excited states that lead to small cascades of tunneling events interrupted by long (Coulomb) blockages, resulting in a noisy current. Since the origin of this effect is completely different to the one discussed above we refer the reader to the work of Belzig and co-workers [8, 13. who have recently discussed this effect for systems restricted to a singly occupied ground state in some detail. The effect can be even more pronounced in more complex systems and ground states with higher charge $q$ [14] as the number of available excited states typically increases with $q$.

In summary, we have discussed the interplay of Coulomb interactions and non-locality in fully symmetric models for a chain of quantum dots. We find superPoissonian shot noise above the sequential tunneling threshold where the electronic current is not suppressed. The Fano factor becomes large in this regime, because the shot noise itself is strongly enhanced. This effect is caused by a competition of "slow" and "fast" transport channels due to the differing spatial structure and total spin of the participating many-body states. We expect the effect to be robust, thus offering a way to study shot noise experimentally in chains of lateral quantum dots in semiconducting heterostructures.

Acknowledgments. We enjoyed interesting and helpful discussions with J. König as well as financial support by the DFG via the Center for Functional Nanostructures.

[1] Ya. M. Blanter and M. Büttiker, Phys. Rep. 336, 1 (2000).

[2] E. V. Sukhorukov, G. Burkard, and D. Loss, Phys. Rev. B 63, 125315 (2001).

[3] B. R. Bulka, Phys. Rev. B 62, 1186 (2000); A. Cottet, W. Belzig, and C. Bruder, Phys. Rev. Lett. 92, 206901 (2004).

[4] G. Michalek and B. R. Bulka, Eur. Phys. J. B 28, 121 (2002).

[5] B. Elattari and S. A. Gurvitz, Phys. Lett. A 292, 289 (2002).

[6] A. Thielmann, M. H. Hettler, J. König, and G. Schön, Phys. Rev. B 68, 115105 (2003); ibid. Phys. Rev. B 71, 045341 (2005).

[7] G. Kiesslich, A. Wacker, and E. Schoell, Phys. Rev. B 68, 125320 (2003).

[8] A. Cottet and W. Belzig, Europhys. Lett. 66, 405 (2004).

[9] A. Thielmann, M. H. Hettler, J. König, and G. Schön, cond-mat/0501534

[10] S. S. Safonov, et al. Phys. Rev. Lett. 91, 136801 (2003).

[11] A. Nauen et al., Phys. Rev. B 70, 033305 (2004).

[12] S. W. Jung, T. Fujisawa, Y. Hirayama, and Y. H. Jeong, Appl. Phys. Lett. 85, 768 (2004).

[13] W. Belzig, cond-mat/0501141

[14] J. Aghassi, A. Thielmann, M. H. Hettler, and G. Schön, in preparation.

[15] J. König, H. Schoeller, and G. Schön, Phys. Rev. Lett. 76, 1715 (1996); J. König, J. Schmid, H. Schoeller, and G. Schön, Phys. Rev. B 54, 16820 (1996).

[16] For the parameters of Fig. 11 right panel, transport in the first plateau is exclusively between $G$ and $S_{1}$. On the second plateau $T_{1}$ and $D_{1}$ join in and obtain equal occupation. Only on the third plateau the bias suffices for the quadruplet $Q$ to be occupied, leading to NDC and enhanced noise. 